\title{
Coloring Fuzzy Circular Interval Graphs
}

\author{
Friedrich Eisenbrand ${ }^{1}$ Martin Niemeier ${ }^{2}$ \\ SB IMA DISOPT \\ $E P F L$ \\ Lausanne, Switzerland
}

\begin{abstract}
Computing the weighted coloring number of graphs is a classical topic in combinatorics and graph theory. Recently these problems have again attracted a lot of attention for the class of quasi-line graphs and more specifically fuzzy circular interval graphs.

The problem is NP-complete for quasi-line graphs. For the subclass of fuzzy circular interval graphs however, one can compute the weighted coloring number in polynomial time using recent results of Chudnovsky and Ovetsky and of King and Reed. Whether one could actually compute an optimal weighted coloring of a fuzzy circular interval graph in polynomial time however was still open.

We provide a combinatorial algorithm that computes weighted colorings and the weighted coloring number for fuzzy circular interval graphs efficiently. The algorithm reduces the problem to the case of circular interval graphs, then making use of an algorithm by Gijswijt to compute integer decompositions.
\end{abstract}

Keywords: fuzzy circular interval graph, circular interval graph, vertex coloring, weighted coloring

${ }_{1}^{1}$ Email: friedrich.eisenbrandeepfl.ch

2 Email: martin.niemeier@epfl.ch 


\section{Introduction}

A weighted $k$ coloring of a graph $G$ with weights $w: V(G) \longrightarrow \mathbb{N}_{0}$ is a multiset of stable sets $S_{1}, \ldots, S_{k} \subseteq V(G)$ such that each vertex $v \in V(G)$ is contained in $w(v)$ many of these stable sets. The weighted coloring number $\chi_{w}(G)$ is the smallest $k$ such that there exist stable sets as above.

A graph is quasi-line if the neighborhood of each of its vertices is the union of two cliques. Chudnovsky and Seymour [2] provided a structural result which states that a connected quasi-line graph is a fuzzy circular interval graph or it is the composition of fuzzy linear interval strips with a collection of disjoint cliques. In particular line-graphs are quasi-line, and thus the weighted coloring problem for quasi-line graphs subsumes the NP-complete edge-coloring problem.

For the subclass of fuzzy circular interval graphs however, one can compute the weighted coloring number in polynomial time, via the equivalence of separation and optimization [5] and recent results of Chudnovsky and Ovetsky [1] and King and Reed [6]. How to compute an optimal weighted coloring for fuzzy circular interval graphs however was still open.

We provide a combinatorial algorithm that addresses this problem. For given $k \in \mathbb{N}$ and a fuzzy circular interval graph of order $n$ with weights $w$, it decides the existence of a weighted $k$ coloring in time $\mathcal{O}\left(n^{3}\right)$ and computes a weighted $k$ coloring in time $\mathcal{O}\left(n^{4}+\operatorname{size}(w)\right)$, where size $(w)$ denotes the binary encoding length of $w$. The weighted coloring number alone can be computed in time $\mathcal{O}\left(n^{2} \operatorname{size}(w)\right)$. The algorithm is based on a reduction to circular interval graphs using an algorithm for maximum $b$-matching and an algorithm of Gijswijt [4] to solve the weighted coloring problem on circular interval graphs.

\section{The structure of quasi-line graphs}

We will review some definitions concerning the structure of quasi-line graphs due to Chudnovsky and Seymour [2].

A graph $G$ is called a circular interval graph, if it can be obtained with the following construction: Let $V(G)$ be a subset of a circle $\mathcal{C}$. Further take a set $\mathcal{I}$ of intervals of the circle $\mathcal{C}$. The set of edges $E(G)$ is defined as follows: Two vertices are adjacent if and only if they are contained in a common interval of $\mathcal{I}$. The pair $(V, \mathcal{I})$ is called a representation of $G$.

A graph $G$ is a fuzzy circular interval graph [2], if the there is a map $\Phi$ from $V(G)$ to a circle $\mathcal{C}$ and a set $\mathcal{I}$ of intervals of $\mathcal{C}$, none including another, such that no vertex of $\mathcal{C}$ is an endpoint of more than one interval so that:

- If two vertices $u$ and $v$ are adjacent, then $\Phi(u)$ and $\Phi(v)$ have a common interval. 
- If two vertices $u$ and $v$ belong to the same interval, which is not an interval with endpoints $\Phi(u)$ and $\Phi(v)$, then they are adjacent.

Fuzzy circular interval graphs provide a generalization of circular interval graphs since adjacencies between nodes mapped to different endpoints of an interval are not specified anymore. For an interval $[p, q] \in \mathcal{I}$, the pair of (nonempty) sets $\left(\Phi^{-1}(p), \Phi^{-1}(q)\right)$ stemming from the preimages of $p$ and $q$ are called fuzzy pairs.

The pair $(\Phi, \mathcal{I})$ is called a representation of $G$. Every fuzzy circular interval graph has a representation whose number of intervals is bounded by $\mathcal{O}(|V(G)|)$ : The fact that no interval is allowed to include another limits the number of irredundant intervals. From now on we assume that the number of intervals is limited by $\mathcal{O}(|V(G)|)$.

Note the special structure of a fuzzy pair $(A, B)$. Both $A$ and $B$ are cliques and every node $v \in V(G) \backslash(A \cup B)$ is either adjacent to every node of $A$ (of $B$ ) or to none of them. In the first case the node $v$ is $A$-complete ( $B$-complete).

The fuzzy pair $(A, B)$ is called nontrivial if $A \cup B$ contains an induced $C_{4}$ subgraph and trivial otherwise. If all fuzzy pairs are trivial, then the graph is a circular interval graph, see e.g. [3].

Lemma 1.1 Given a fuzzy circular interval graph $G$ and a representation, if every fuzzy pair of $G$ w.r.t. that representation is trivial, then $G$ is a circular interval graph.

\section{The coloring algorithm}

An application of a result by Gijswijt [4] shows that so called integer decompositions, see e.g. [8], in the stable set polytope of circular interval graphs can be computed efficiently. Since integer decompositions in stable set polytopes essentially are weighted colorings, this immediately gives:

Theorem 2.1 [4] Given a circular interval graph $G$ with weights $w$, for every $k \in \mathbb{N}$ we can decide if a weighted $k$ coloring of $(G, w)$ exists in time $\mathcal{O}\left(|V(G)|^{2}\right)$.

A weighted $k$ coloring can be computed in time $\mathcal{O}\left(|V(G)|^{2}+\operatorname{size}(w)\right)$. The number of different stable sets in the coloring is bounded by $\mathcal{O}(|V(G)|)$.

Our coloring algorithm for fuzzy circular interval graphs will reduce to the case of circular interval graphs by transforming the input graph $G$ and its weights $w$ to a circular interval graph $G^{*}$ with weights $w^{*}$ such that the coloring number is preserved, i.e. $\chi_{w}(G)=\chi_{w^{*}}\left(G^{*}\right)$. Then it makes use of the algorithm from the theorem above to obtain a coloring of $G^{*}$, which finally gets transformed to a coloring of $G$. 
Recent results of Chudnovsky and Ovetsky [1] and of King and Reed [6] give algorithms to reduce fuzzy circular interval graphs to circular interval graphs preserving the (nonweighted) coloring number. By replacing each node $v \in V(G)$ of $G$ with a clique of size $w(v)$ one could reduce to this simpler case. However this would result only in a pseudopolynomial algorithm.

\section{The reduction}

We show how to replace a single fuzzy pair by a nontrivial one preserving the coloring number. Applying the construction succesively for every fuzzy pair, with Lemma 1.1 we get a circular interval graph $G^{*}$ with weights $w^{*}$ as required above.

Consider a fuzzy circular interval graph $G$ with weights $w$ and a fuzzy pair $(A, B)$ in $G$. Let $V^{\circ}:=V(G) \backslash(A \cup B)$. Then the reduced graph $\left(G^{\prime}, w^{\prime}\right)$ is defined as follows:

$$
\begin{aligned}
& V\left(G^{\prime}\right):=V^{\circ} \cup\left\{a_{0}, a_{1}, b_{0}, b_{1}\right\}, \\
& E\left(G^{\prime}\right):=\left.E(G)\right|_{V^{\circ}} \cup\left\{\left\{v, a_{0}\right\},\left\{v, a_{1}\right\}: v \in V^{\circ} A \text {-complete }\right\} \\
& \cup\left\{\left\{v, b_{0}\right\},\left\{v, b_{1}\right\}: v \in V^{\circ} B \text {-complete }\right\} \\
& \cup\left\{\left\{a_{0}, a_{1}\right\},\left\{b_{0}, b_{1}\right\},\left\{a_{0}, b_{1}\right\},\left\{a_{1}, b_{1}\right\},\left\{a_{1}, b_{0}\right\}\right\} \\
& w^{\prime}(v):= \begin{cases}\alpha & \text { if } v=a_{0} \text { or } v=b_{0} \\
w(A)-\alpha & \text { if } v=a_{1} \\
w(B)-\alpha & \text { if } v=b_{1} \\
w(v) & \text { else }\end{cases}
\end{aligned}
$$

A similar construction is used in independent work of Oriolo, Pietropaoli and Stauffer [7] who designed an efficient recognition algorithm for fuzzy circular interval graphs.

We next specify $\alpha$. The sets $A$ and $B$ together with the complement of the edges of $G[A \cup B]$ define a bipartite graph $H$. If a stable set $S$ of $G$ has two vertices in $A \cup B$, then those two vertices are connected by an edge in $H$. Observe that in a weighted coloring of $G$, the maximum number of stable sets containing two vertices of $A \cup B$ is given by the size of a largest $b$-matching in $H$, where the labels on the nodes $v \in A \cup B$ are given by the coloring demand $w(v)$. The number $\alpha$ from the reduction above is the size of a largest $b$-matching in $H$.

It is straightforward to verify that $G^{\prime}$ is still a fuzzy circular interval graph. Moreover if $(A, B)$ was nontrivial, then the number of nontrivial fuzzy pairs has been reduced by one since $\left(\left\{a_{0}, a_{1}\right\},\left\{b_{0}, b_{1}\right\}\right)$ is trivial. We show that the construction preserves the weighted coloring number. 
Lemma 2.2 With the reduction above one has $\chi_{w}(G)=\chi_{w^{\prime}}\left(G^{\prime}\right)$.

Proof If is sufficient to show that every weighted $k$ coloring of $\left(G^{\prime}, w^{\prime}\right)$ gives rise to a weighted $k$ coloring of $(G, w)$ and vice versa. We only sketch the proof here. Let $A^{\prime}=\left\{a_{0}, a_{1}\right\}$ and $B^{\prime}=\left\{b_{0}, b_{1}\right\}$.

Observe that by construction, for each weighted coloring $S_{1}^{\prime}, \ldots, S_{k}^{\prime}$ of $\left(G^{\prime}, w^{\prime}\right)$, the number $\mu$ of stable sets containing two points of $A^{\prime} \cup B^{\prime}$ is bounded by $\alpha$. Therefore we can assign an edge $e$ of a maximum matching in $H$ to each of these sets. Replacing $\left\{a_{0}, b_{0}\right\}$ by $e$ gives a stable set in $G$. For the remaining $w(A)-\mu$ sets containing a node of $A^{\prime}$ but not of $B^{\prime}$ we assign nodes $a \in A$ such that each node is covered $w(a)$ times in total. The same we do for the remaining $w(B)-\mu$ sets containing a node of $B^{\prime}$ but not of $A^{\prime}$. All sets not considered so far are left unchanged. The resulting sets form a $k$-coloring of $(G, w)$.

The vice versa transformation works analogous and we skip the details.

To compute the reduced graph $\left(G^{\prime}, w^{\prime}\right)$, we need time $\mathcal{O}\left(|V(G)|^{2}\right)$ to compute the auxiliary graph $H$ and encode the output, and $\mathcal{O}\left(|A \cup B|^{3}\right)$ to compute the maximum $b$-matching in $H$ using a max $s-t$-flow algorihm, see e.g. [8]. Thus the running time for a single reduction step is bounded by $\mathcal{O}\left(|V(G)|^{2}+|A \cup B|^{3}\right)$.

From a representation of the graph we can easily compute the fuzzy pairs. Their number $l$ is bounded by the number of intervals in the representation, which is $\mathcal{O}(|V(G)|)$. Let $\left(A_{i}, B_{i}\right), i=1, \ldots, l$ denote the fuzzy pairs of $G$. Invocation of the reduction for each fuzzy pair $\left(A_{i}, B_{i}\right)$ accumulates a total running time of $\mathcal{O}\left(l \cdot|V(G)|^{2}+\sum_{i=1}^{l}\left|A_{i} \cup B_{i}\right|^{3}\right)=\mathcal{O}\left(|V(G)|^{3}\right)$. With Theorem 2.1 we get:

Theorem 2.3 Given a fuzzy circular interval graph $G$ with weights $w$ and a representation, in time $\mathcal{O}\left(|V(G)|^{3}\right)$ for each $k \in \mathbb{N}$ one can decide if a weighted $k$ coloring of $(G, w)$ exists.

One can also find $\chi_{w}(G)$ in time $\mathcal{O}\left(|V(G)|^{2} \cdot \operatorname{size}(w)\right)$ by reducing to circular interal graphs and then doing binary search on $[1, w(V)]$ using Gijswijts algorithm.

To actually compute a weighted $k$ coloring of $(G, w)$, we have to transform the $k$ coloring of $\left(G^{*}, w^{*}\right)$ generated by Gijswijts algorithm to a $k$ coloring of $(G, w)$. We use the transformation given in Lemma 2.2 repeatedly. Let $n=|V(G)|$. Straightforward analysis of the running time needed for each transformation gives a pseudopolynomial bound of $\mathcal{O}\left(k \cdot n+n^{2}+|A \cup B|^{3}\right)$.

We sketch how to get the polynomial bound. In fact the $k$ coloring computed by Gijswijt's algorithm is given in a compact formulation $\left(\lambda_{1}, S_{1}^{\prime}\right), \ldots,\left(\lambda_{\beta}, S_{\beta}^{\prime}\right)$ with $\lambda_{i} \in \mathbb{N}$ and $\sum_{i=1}^{\beta} \lambda_{i}=k$, meaning that the stable set $S_{i}^{\prime}$ should appear $\lambda_{i}$ times. The number $\beta$ of different stable is sets $\mathcal{O}(n)$. Also the $b$-matching computed for $H$ is 
given in a similar compact formulation. Trivially the number of different edges in the matching is bounded by $|A \cup B|^{2}$. Now if $S_{i}^{\prime}$ is a set containing both $a_{0}$ and $b_{0}$ and we have an edge $e$ appearing $\gamma$ many times, we can transform $\min \left\{\lambda_{i}, \gamma\right\}$ sets simultaneously. We can speedup the transformation of the other sets similarly.

If the matching edges are assigned arbitrarily, the resulting running time is $\mathcal{O}\left(\left(\beta+|A \cup B|^{2}\right) \cdot n+n^{2}+|A \cup B|^{3}\right)$. Moreover, the number of different independent sets in the transformed coloring is bounded by $\beta+|A \cup B|^{2}$. Applying this transformation for each fuzzy pair, the number of different stable sets never exceeds $\mathcal{O}\left(|V(G)|^{2}\right)$ and there are at most $n$ transformations needed. Thus the total running time can be bounded with $\mathcal{O}\left(|V(G)|^{4}\right)$. With Theorem 2.1 we get the following.

Theorem 2.4 Given a weighted fuzzy circular interval graph $(G, w)$ and a representation. For every $k \in \mathbb{N}$, in time $\mathcal{O}\left(|V(G)|^{4}+\operatorname{size}(w)\right)$ one can compute a weighted $k$ coloring of $(G, w)$.

\section{References}

[1] M. Chudnovsky and A. Ovetsky. Coloring quasi-line graphs. J. Graph Theory, 54(1):41-50, 2007.

[2] M. Chudnovsky and P. D. Seymour. The structure of claw-free graphs. In Surveys in Combinatorics, pages 153-171, 2005.

[3] F. Eisenbrand, G. Oriolo, G. Stauffer, and P. Ventura. The stable set polytope of quasiline graphs. Combinatorica, 28(1):45-67, 2008.

[4] D. Gijswijt. Integer decomposition for polyhedra defined by nearly totally unimodular matrices. SIAM J. Discret. Math., 19(3):798-806, 2005.

[5] M. Grötschel, L. Lovász, and A. Schrijver. Geometric algorithms and combinatorial optimization, volume 2 of Algorithms and Combinatorics. Springer-Verlag, Berlin, second edition, 1993.

[6] A. D. King and B. Reed. Asymptotics of the chromatic number for quasi-line graphs. Electronic Notes in Discrete Mathematics, 29:327 - 331, 2007. European Conference on Combinatorics, Graph Theory and Applications.

[7] G. Oriolo, U. Pietropaoli, and G. Stauffer. On the recognition of fuzzy circular interval graphs. to appear, 2009.

[8] A. Schrijver. Combinatorial Optimization - Polyhedra and Efficiency. Springer, 2003. 